\title{
ON SURFACES IN SPACES OF FOUR AND FIVE DIMENSIONS
}

\author{
BY B. C. WONG
}

It is known that an algebraic surface in 4-space has four essential characteristics in terms of which all its other characteristics can be expressed. Severi* considers as essential the following: $n$, the order of the surface; $a$, the order of the tangent cone of its projection in a 3 -space; $t$, the number of its apparent triple points; and $n^{\prime}$, the number of 3 -spaces that can be constructed tangent to it and passing through a given point.

In order to know anything about a surface in 4 -space, it is, then, necessary to know its four essential characteristics. Of course, $n$ may always be assumed. Unless some independent means be found whereby the other three characteristics, $a, t$, $n^{\prime}$ can be calculated, we cannot know very much about the surface. It is our purpose in this paper to present a method for the independent determination of these three characteristics.

Let $F^{\prime n}$ denote a surface of order $n$ in 4 -space, and if the surface is the projection of a surface of the same order in 5-space, let $F^{n}$ denote the latter. We introduce three other characteristics of $F^{\prime n}: b$, the order of the cone of lines passing through a general point in $S_{4}$ and meeting $F^{\prime n}$ twice; $j$, the number of tangent lines of $F^{\prime n}$ passing through a given point; and $d$, the number of improper double points $\dagger$ on $F^{\prime n}$.

The seven characteristics are connected by the following relations :

$$
\begin{aligned}
& a+2 b=n(n-1), \quad j+2 d=n(n-1)-a, \\
& j=\frac{1}{4}\left[a(3 n-4)-n(n-1)(n-2)+6 t-2 n^{\prime}\right], \\
& d=\frac{1}{8}\left[n(n-1)(n+2)-3 n a-6 t+2 n^{\prime}\right] .
\end{aligned}
$$

* Severi, Intorno ai punti doppi impropri di una superficie generale dello spazio a quattro dimensioni, e a'suoi punti tripli apparenti, Rendiconti di Palermo, vol. 15 (1901), pp. 33-51.

† An improper double point $Q$ is one such that an $S_{3}$ through it meets the surface in a curve with an actual double point at $Q$ and of the same deficiency as that of a general 3-space section of the surface.

‡ Severi, loc. cit., pp. 34-36. 
From these relations we see that, $n$ being assumed, if $a, t$, $n^{\prime}$ are known, $j$ and $d$ are determined. On the other hand, if we know $t$ and any two of the three quantities $a, j, d$, we can calculate $n^{\prime}$. Our method will, however, enable us to determine directly all the four quantities $a, j, d$, and $t$.

Consider a surface $F^{n}$ of order $n$ in $S_{5}$ and let it be the complete intersection of three hypersurfaces $V_{4}{ }^{\lambda}, V_{4}^{\mu}, V_{4}{ }^{\nu}$ of orders $\lambda, \mu, \nu$ respectively. We shall represent $F^{n}$ symbolically* by means of $n=\lambda \mu \nu$ triads $(x, y, z)$ whose elements $x, y, z$ are to take on all the integral values from 1 to $\lambda, \mu, \nu$ respectively. Thus, the surface $F^{8}$ common to three hyperquadric surfaces will be represented by the eight triads $(1,1,1),(2,1,1),(1,2,1)$, $(1,1,2),(1,2,2),(2,1,2),(2,2,1),(2,2,2)$. If one of the hypersurfaces, say $V_{4}{ }^{\nu}$, is a hyperplane $S_{4}$, the surface is a 4 -space $F^{\prime n}$ of order $n=\lambda \mu$ lying in $S_{4}$ and will be represented by the $\lambda \mu$ triads $(x, y, 1)$.

Any triad taken alone in this representation of $F^{n}$ represents a plane forming a part of $F^{n}$. Any pair of triads with two corresponding elements alike, as $(1,1,1),(1,1,2)$ or $(1,2,3),(4,2,3)$ represents a quadric surface or a pair of planes with a line in common. If two of the corresponding elements are different, as $(1,1,1),(1,2,2)$ or $(1,2,3),(4,1,3)$, we have a pair of planes intersecting in a point; but if all the three corresponding elements are different, the two planes have no point in common.

Now we determine the characteristic $a$. It is not difficult to see that $a$ is the order of the hypersurface formed by the $\infty^{1}$ tangent 3 -spaces of $F^{n}$ that pass through a given plane $\alpha$ in $S_{5}$. Consider a quadric surface $F^{2}$ given in a general $S_{3}$ of $S_{5}$. Since $S_{3}$ has only a point in common with $\alpha$, the $\infty^{1}$ tangent 3 -spaces of $F^{2}$ passing through $\alpha$ form a hypersurface $V_{4}^{2}$ of order 2. If $F^{2}$ degenerates into two planes which must have a line in common, $V_{4}{ }^{2}$ degenerates into the hyperplane determined by $\alpha$ and the line, counted twice. From this we infer that, in general, if $F^{n}$ contains $N$ double lines, the order of the hypersurface of tangent 3 -spaces passing through a given plane is $a-2 n$. Suppose $F^{n}$ be composed entirely of planes, $n=\lambda \mu \nu$ in

* B. C. Wong, On the number of apparent triple points of surfaces in space of jour dimensions, this Bulletin, vol. 35 (1929), pp. 339-343; and On the number of apparent multiple points of varieties in hyperspace, this Bulletin. vol. 36 (1930), pp. 102-106. 
number. The number $N$ of double lines on this degenerate $F^{n}$ is equal to the number of pairs of triads in the representation of $F^{n}$ with two corresponding elements alike. As $F^{n}$, degenerated in this way, cannot have proper tangent 3 -spaces, we have $a-2 N=0$ or $a=2 N$.

To find $N$, we notice that, for all the integral values of $x$ and $y$ from 1 to $\lambda$ and $\mu$ respectively, we have $\lambda \mu \nu(\nu-1) / 2$ pairs of triads of the nature described above. Permuting $\lambda, \mu, \nu$ and adding the results, we have $N=\lambda \mu \nu(\lambda+\mu+\nu-3) / 2$ and, therefore $a=\lambda \mu \nu(\lambda+\mu+\nu-3)^{*}$ is the required formula for $a$.

Now we proceed to determine $j$. Consider a pair of triads with two corresponding different elements representing a pair of planes having a point in common. Any plane through this point and through a given line $g$ in $S_{5}$ is to be considered a tangent plane of $F^{n}$ through $g$, counted doubly. Any plane through $g$ and tangent to a non-degenerate $F^{n}$ gives rise to a line through a point in an $S_{4}$ and tangent to the projection $F^{\prime n}$ in $S_{4}$. Then, the number of tangent planes of $F^{n}$ passing through $g$ which is equal to the number of tangent lines of $F^{\prime n}$ passing through a given point in $S_{4}$ is the number $j$ we are seeking. But if $F^{n}$ be decomposed into $n$ planes, there will be no proper tangent planes. Through a given line pass a certain number, $N^{\prime}$, of planes each passing through a point in which two planes of $F^{n}$ as represented above intersect. As each such plane counts twice as a tangent plane of $F^{n}$, we have $j=2 N^{\prime}$.

To calculate $N^{\prime}$, we find that, for a fixed value of $x$, the number of pairs of triads such that the $y$-and z-elements of one of the triads in any pair are different from the corresponding elements of the other triad of the same pair is given by the expression $\mu \nu(\mu-1)(\nu-1) / 2$. By allowing $x$ to vary from 1 to $\lambda$, we have $\lambda \mu \nu(\mu-1)(\nu-1) / 2$. Permuting $\lambda, \mu, \nu$ and adding, we have the total number $N^{\prime}$ of the desired pairs of triads in the representation of $F^{n}$. Therefore, twice this number is

$$
j=\lambda \mu \nu(\mu-1)(\nu-1)+(\nu-1)(\lambda-1)+(\lambda-1)(\mu-1) .
$$

To derive a formula for $d$, we consider a pair of triads with

* This formula can be obtained by the same method as that employad by Salmon to determine the rank of a 3-space curve which is the complete intersection of two surfaces. See Salmon, Analytic Geometry of Three Dimensions, 6th ed., vol. I, Paragraphs 342, 343. 
corresponding elements all unlike. The planes they represent are all skew and there is just one line passing through a given point and incident with these planes. Such a line gives rise to an improper double point on the projection $F^{\prime n}$ of $F^{n}$ in $S_{4}$. Hence, the total number of pairs of triads in the representation of $F^{n}$ of the nature just described is the number $d$. As each of the $\lambda$ integral values of $x$ is to be combined with each of the $\mu$ integral values of $y$ and also with each of the $\nu$ integral values of $z$, we have

$$
d=k \lambda \mu \nu(\lambda-1)(\mu-1)(\nu-1),
$$

where $k$ is a yet unknown constant. By actual trial for the case $\lambda=\mu=\nu=2$, we find $d=4$ and, therefore, $k=1 / 2$. Then

$$
d=\frac{1}{2} \lambda \mu \nu(\lambda-1)(\mu-1)(\nu-1)
$$

is the required formula.

It is to be noted that if one of the three hypersurfaces, say $V_{4} \nu$, is a hyperplane $S_{4}$, that is, $\nu=1$, we have

$$
d=0, j=\lambda \mu(\lambda-1)(\mu-1) .
$$

That is, the surface $F^{\prime n}$ of order $n^{\prime}=\lambda \mu$ which is the complete intersection of two hypersurfaces $V_{3}^{\lambda}, V_{3}^{\mu}$, in $S_{4}$ cannot have improper double points and cannot be the projection of a surface of the same order in $S_{5}$. The number of its tangent lines passing through a given point in $S_{4}$ is always twice the order of the cone of lines passing through a given point and incident with the surface twice.

It remains to determine $t$. For this purpose we consider triples of triads in the representation of $F^{n}$. The triples that we need are of four types. Those of type I each represent three planes lying in a 4 -space. Two corresponding elements of the triads of such a triple must be different, as $(1,1,1),(1,2,2)$, $(1,3,3)$ or $(1,1,1),(3,1,2),(2,1,4)$. Those of type II each represent three planes lying two by two in three 4 -spaces. Every pair of triads of such a triple must have one and only one element in common, as for example, $(1,1,1),(1,2,2),(2,1,2)$ or $(1,2,1),(1,4,3),(2,2,3)$ or $(1,2,3),(1,4,2),(3,2,2)$. Type III consists of those triples each of which represents three planes such that two of them lie in a 4-space and the third may or may not lie in another 4-space with one of them. Two of the triads of 
such a triple must have one element in common and the third may or may not have one of the remaining elements in common with one of them. Examples are $(1,1,1),(1,2,2),(2,3,2) ;(1,1,1)$, $(1,2,3),(2,3,2)$. Type IV consists of all those triples the triads of each of which have all their corresponding elements different, as $(1,1,1),(2,2,2),(3,3,3)$ or $(1,2,3),(2,3,1),(3,1,2)$. The planes represented by such a triple are all skew.

The three planes represented by any triple belonging to any of these four types are such that through a given line in $S_{5}$ not incident with them passes just one plane meeting them each in a point. Such a plane gives rise to an apparent triple point on the projection $F^{\prime n}$ of $F^{n}$ in $S_{4}$. Hence, to find $t$ is to find the sum of the numbers $T, T^{\prime}, T^{\prime \prime}, T^{\prime \prime \prime}$ of the triples of points belonging to the four types, respectively, obtained from the representation of $F^{n}$.

Reasoning in a manner analogous to that in which the formulas for $a, j, d$ are derived, we obtain the following, which can be verified without difficulty:

$$
\begin{aligned}
T= & \frac{1}{6} \lambda \mu \nu[(\mu-1)(\mu-2)(\nu-1)(\nu-2) \\
& +(\nu-1)(\nu-2)(\lambda-1)(\lambda-2) \\
& +(\lambda-1)(\lambda-2)(\mu-1)(\mu-2)], \\
T^{\prime}= & \lambda \mu \nu(\lambda-1)(\mu-1)(\nu-1), \\
T^{\prime \prime}= & \frac{1}{2} \lambda \mu \nu(\lambda-1)(\mu-1)(\nu-1)(\mu \nu+\nu \lambda+\lambda \mu-12), \\
T^{\prime \prime \prime}= & \frac{1}{6} \lambda \mu \nu(\lambda-1)(\lambda-2)(\mu-1)(\mu-2)(\nu-1)(\nu-2) .
\end{aligned}
$$

Then we have $t=T+T^{\prime}+T^{\prime \prime}+T^{\prime \prime \prime}$.

So far, we have dealt with surfaces which are complete intersections of hypersurfaces. It remains to say a few words concerning those surfaces which are partial intersections. For $n<\lambda \mu \nu$, we still have $n$ triads in the symbolic representation of $F^{n}$. These $n$ triads must be such that any of them has two corresponding elements in common with at least one other element. Every non-degenerate $F^{n}$ has its own representation and every arrangement or group of $n$ triads whose elements satisfy the above requirement represents a non-degenerate $F^{n}$ in $S_{5}$. Given an $F^{n}$, we find the characteristics $a, j, d, t$ of its projection $F^{\prime n}$ in $S_{4}$ by counting the numbers of the respective pairs and triples of triads in its representation of the nature already explained. Let us illustrate. 
A surface $F^{4}$ of order 4 in $S_{5}$ is of one of three types, represented respectively by the triads

According to the principle explained above, it is not difficult to see that an $F^{4}$ represented by (a) is a Veronese quartic surface in $S_{5}$. Its projection $F^{\prime n}$ in $S_{4}$ has one $\left(t=T^{\prime}=1\right)$ apparent triple point, for there is only one triple of triads given by [2], [3], [4] belonging to one of the four types explained above, indeed belonging to type II ; and has no $(d=0)$ improper double point, for there is no pair of triads with corresponding elements all unlike. The projection of $F^{\prime \prime}$ in an $S_{3}$ is a Steiner's quartic surface and has one triple point and three double lines. As the number of pairs of triads with two corresponding elements different is 3 , given by [2], [3]; [3], [4]; [2], [4], there are $j=2 \cdot 3=6$ pinch-points on this projected surface in $S^{3}$; and as the number of pairs of triads with two corresponding elements alike is also 3 , given by [1], [2]; [1], [3]; [1], [4], the tangent cone is also of order $a=2 \cdot 3=6$.

Examining the four triads of (b) and those of (c) in a similar manner, we find that an $F^{4}$ represented by (b) has for projection in $S_{4}$ an $F^{\prime n}$ for which $a=6, d=1, j=4$, and $t=0$. Its projection in $S_{3}$ is a ruled quartic surface with a double twisted cubic curve upon which lie four pinch-points and no triple point. We also find that the surface represented by (c) is already a 4 -space surface, the Segre quartic surface, being the complete intersection of three hypersurfaces in $S_{5}$ one of which is a hyperplane and the other two are of order 2 . The formulas above derived apply and we find $a=8, j=4, d=0, t=0$. Its projection in $S_{3}$ has a double conic on which are four pinchpoints.

For other values of $n$ we proceed in the same manner. Of course, when $n$ is large, this process of counting is laborious but the desired characteristics can invariably be found.

The University of California 